\title{
investigación por métodos ultrasónicos de/ estado de deterioro del hormigón de una estructura sometida a fuego
}

ANSELMO GARAY, Lodo. en Física

\begin{abstract}
sinopsis
Cuando un edificio con estructura de hormigón arınado sufre un incendio, los elementos de aquélla quedan afectados en una profundidad variable con la duración y las temperaturas máximas alcanzadas.

En el presente artículo se presenta un método de tipo no destructivo que, partiendo de las mediciones de los tiempos de transmisión de haces ultrasónicos a través de las piezas afectadas, permite calcular la profundidad media de calcinación en dichos elementos.

Se concluye el artículo con las consideraciones e indicaciones oportunas para la confección de un programa de cálculo electrónico que permita tratar la gran masa de datos procedentes del análisis de un incendio real de medianas proporciones.
\end{abstract}

\section{INTRODUCCION}

Los incendios son accidentes relativamente frecuentes en nuestros días. Si bien se suelen dar más en edificaciones donde abundan los materiales combustibles, tampoco se libran de ellos los grandes y pequeños edificios dedicados a las diversas actividades humanas, particulares o industriales con estructuras de hormigón armado.

Este material, como todos sabemos, si bien tiene algunas buenas cualidades a efectos de resistencia frente al fuego, son siempre limitadas y las conserva un cierto tiempo también limitado, dependiente de varios factores entre los que figuran como decisivos el grado de exposición al fuego.

Ahora bien, el deterioro de las cualidades mecánicas del hormigón no se debe sólo a la destrucción por calcinación de sus componentes químicos, sino también a la destrucción parcial de ciertas zonas superficiales por fisuras producidas por efecto del choque térmico al que se suele someter dichas zonas durante la extinción del incendio con chorros de agua fría.
Ello produce contracciones bruscas y desiguales que dan lugar en el elemento de hormigón a toda una red de fisuras o microfisuras, que le afectan hasta una cierta profundidad.

Pero, además, a causa de las fuertes dilataciones de que son objeto las armaduras, la capa de recubrimiento de las mismas suele saltar total o parcialmente o, al menos, queda mermada en gran parte la adherencia entre ambas, facilitándose así el fácil desprendimiento de aquélla y perjudicando el correcto funcionamiento de la armadura.

Por otra parte, los áridos gruesos más externos, en contacto casi directo con el fuego, se rompen o cuartean, coadyuvando a la acción destructiva en el mortero superficial, lo que. junto con la fisuración apuntada anteriormente, asegura la disgregación práctica de las capas de recubrimiento si el tiempo de exposición a las altas temperaturas alcanza un cierto límite y, sobre todo si se somete a continuación la pieza a chorros de agua fría, como es usual tan pronto aparecen en escena los equipos de extinción. De hecho, se ha 


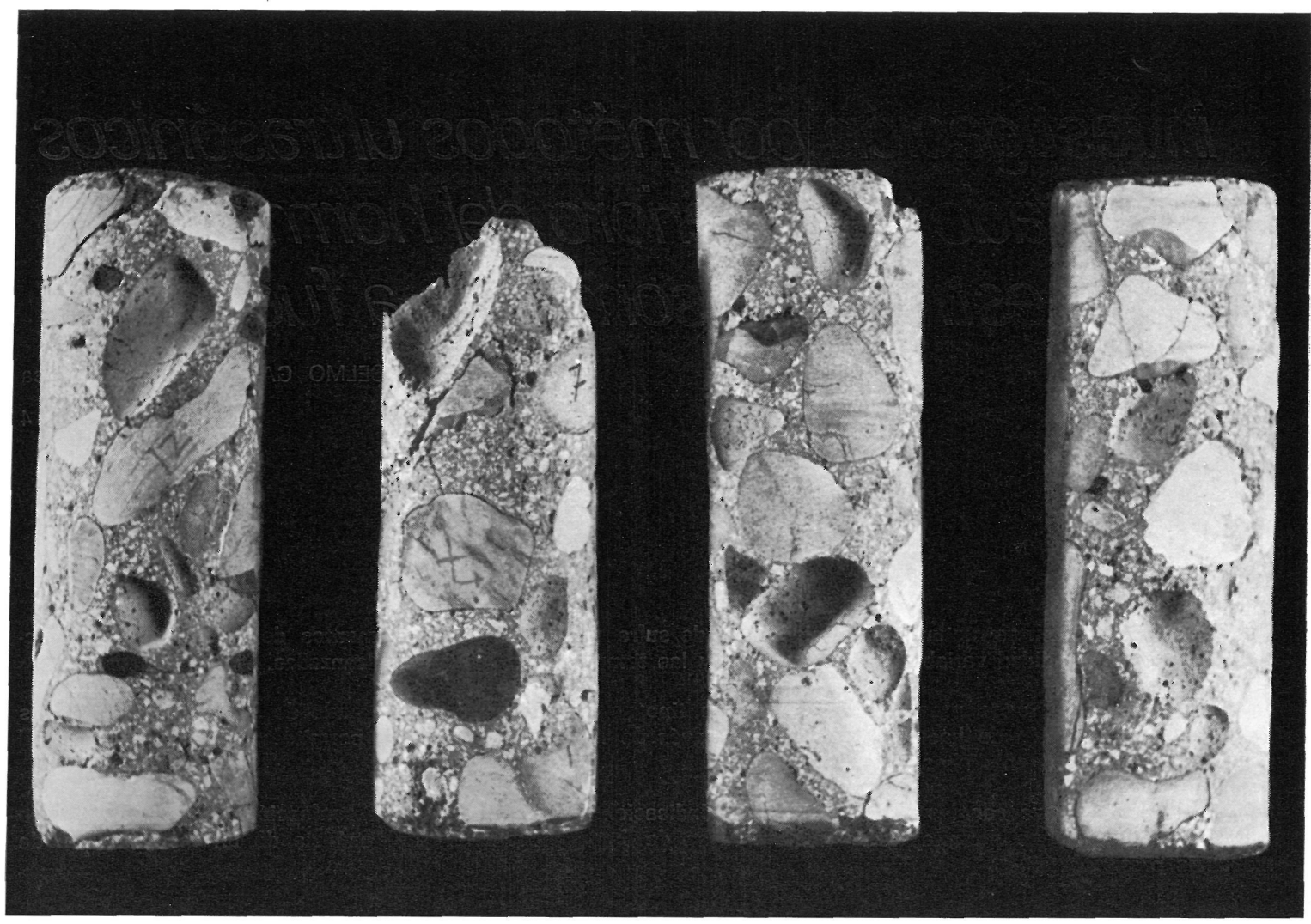

Fig. 1. Probetas sometidas a $700^{\circ} \mathrm{C}$ y posterior choque térmico con agua a $10^{\circ} \mathrm{C}$.

comprobado que resulta preferible en ciertas condiciones (poco material combustible por arder, temperaturas excesivamente altas, etc.) prescindir del empleo de agua en la extinción.

Cuando uno de estos incendios ocurre en cualquier edificio con estructura de hormigón, es usual encargar a un equipo técnico apropiado la tarea de tratar de encontrar, por los medios que sean, el modo de obtener toda la información posible sobre el estado resistente del material de estructuras (hormigón) después de la acción agresiva del fuego, con vistas a una esti. mación, lo más realista posible, de la resistencia residual en los elementos resistentes, que nos permita proyectar el tipo de reparación o refuerzo más adecuado.

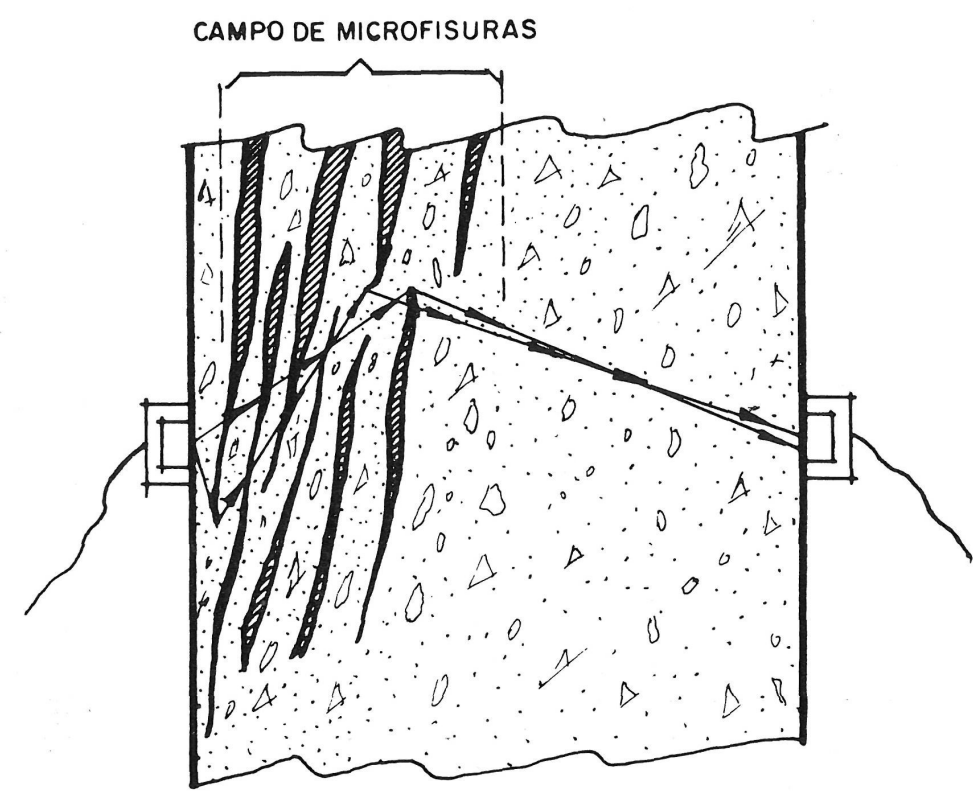

Fig. 2 a. Paso de un haz ultrasónico por un campo de microfisuras 


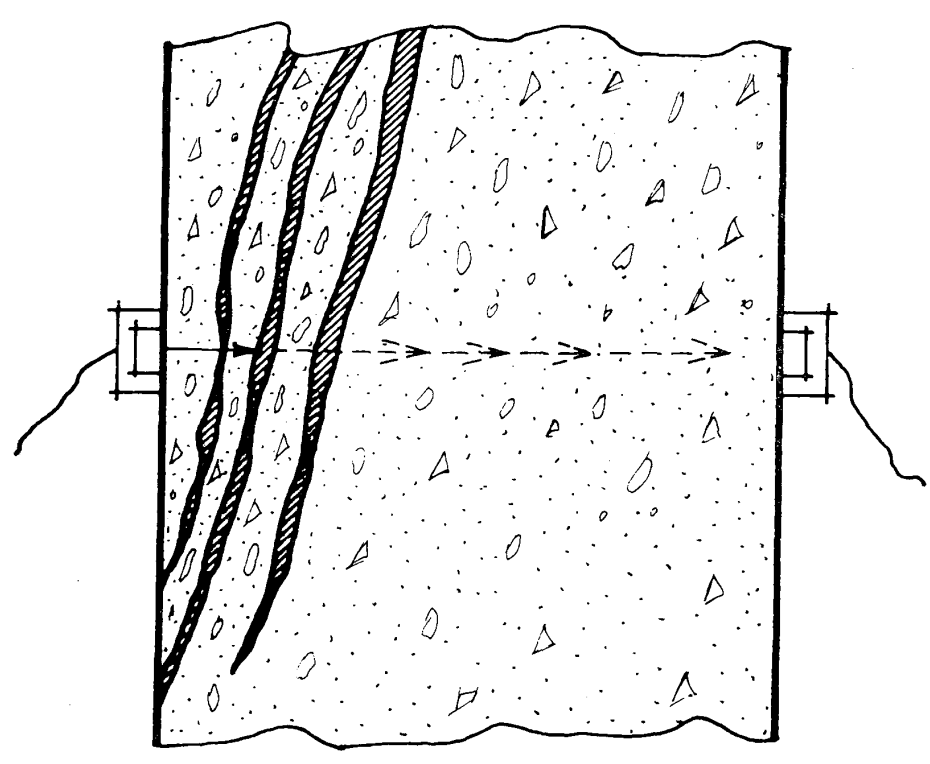

Fig. 2b. Interrupeión de un haz ultrasónico por un campo
y/o espesor.
Hasta ahora sólo se han sugerido, que sepamos, ensayos informativos por muestreo incompleto de la estructura dañada, a base de extracción de testigos de ciertas y limitadas zonas de las mismas, para después, mediante algunos «test» o bien de tipo químico (uso de la fenolftaleína para indicación del $\mathrm{pH}$ del mortero), o bien de tipo mecánico (corte de los testigos en rodajas y posterior ensayo de las mismas a compresión en prensa, con vistas a la determinación de las resistencias parciales según la profundidad) * determinar de algún modo el estado resis. tente residual de los elementos ensayados y extrapolar luego, con el peligro que eso entraña, los resultados al resto de la zona afectada.

En vista de la escasez de ensayos coniables para atacar este tipo de problemas, pensamos en la posibilidad del uso de ultrasoni. dos, de una forma práctica, para la detección de este tipo de daños que, como decíamos al

\footnotetext{
* Si se contemplan las dificultades inherentes a estos métodos, tal como extraer en un buen estado un testigo de una región de hormigón calcinado cortarlo después en rodajas, relativamente finas, si se quiere que sea eficaz el ensayo, sin que se destruyan éstas, romperlas a compresión con garantías y utilizar finalmente, con rigor un resultado procedente de una probeta con una relación $h / D$, necesariamente baja, estos métodos de determinación de daños se nos antojan verdaderamente impracticables.
}

principio, no se limitan a un mero deterioro de las cualidades elásticas y resistentes del material, sino que se suelen acompañar por todo un complejo de fisuras y microfisuras de profundidad variable, como ya dijimos antes.

El efecto de cuarteo y destrucción superficial ha podido ser constatado por nosotros mismos, sometiendo cierto número de probetas a temperaturas variables entre $250^{\circ} \mathrm{C}$ y $700^{\circ} \mathrm{C}$ y rociándolas a continuación lentamente con agua fría. Se comprobó visualmente todos los efectos destructivos anterior. mente detallados (fig. 1).

Por todas estas razones, en la investigación de los daños por el método que proponemos y que son ocasionados en capas más profun. das del hormigón, habrá que prescindir de estas zonas superficiales por picado de las mismas (lo que se desprenda fácilmente con el martillo) para evitar todas las perturbaciones que su presencia pudiera causar en las medidas subsiguientes.

Estos daños profundos se reducen, en general, a la degradación de las cualidades mecá. nico-resistentes del material, por calcinación de sus componentes, pudiendo ir acompañado en muchos casos de la presencia de microfisuras que alcanzan estas zonas más profundas. Suelen producirse en planos sensiblemente paralelos a las caras externas de la pieza y se ponen de manifiesto, durante la 
exploración de ésta con ultrasonido, bien porque interrumpen totalmente el haz de exploración o bien porque alargan anómalamente el camino geométrico de propagación obligando a la onda a marchar por zonas próximas no fisuradas (fig. 2). No obstante, estas conclusiones deberán sacarse en cada caso tras una concienzuda interpretación de los tiempos de propagación y otras circuns. tancias de las medidas en obra.

\section{FUNDAMENTOS DEL METODO}

El fundamento del método que proponemos, que al utilizar solamente medidas de tiempo de propagación ultrasónica resulta así completamente NO DESTRUCTIVO, tiene la ventaja de poderse aplicar a cuantos elemen. tos estructurales sea necesario y cuantas secciones de los mismos deseemos.

Su fundamento consiste en usar como parámetro definidor del grado de deterioro del material, los tiempos de tránsito de impulsos ultrasónicos a través de la zona de ensayo.

En efecto: la velocidad de las ondas sonoras longitudinales a través de un sólido viene ligada con las constantes elásticas de éste por:

$$
c_{l}=\sqrt{\frac{E}{\varrho} \cdot \frac{1-\mu}{(1+\mu)(1-2 \mu)}}=\sqrt{\frac{E}{\varrho} \cdot \varphi(\mu)}
$$

Donde:

$E=$ Módulo de Young.

$\varrho=$ Densidad

$\mu=$ Coeficiente de Poisson.

$c_{l}=$ Velocidad de las ondas longitudinales.

Aquí se ve que al decrecer $E$ lo hace también $c_{l} \mathrm{y}$, aproximadamente, a la par que $\sqrt{E}$ si se desprecia la pequeña variación que puede introducir el descenso de $\varrho$ y el pequeño aumento de $\varphi(\mu)$ con las cualidades elásticas del material.

Según esto, la rápida disminución del módulo elástico $E$, con la destrucción gradual por calcinación de los compuestos cristalinos hidratados que forman el hormigón, se traduce necesariamente en una disminución de la velocidad de propagación, $c_{l}$, de las ondas sónicas o ultrasónicas longitudinales que atraviesen el material.
Por otra parte, numerosos autores han comprobado la existencia de una estrecha correlación entre la velocidad de la onda longitudinal y la resistencia a compresión, $\sigma$, del hormigón.

Nosotros mismos hemos tenido ocastón de verificar dicha correlación en numerosos controles de obras de hormigón armado, en las que hemos realizado exploraciones ultrasónicas in situ de algunos de sus elementos, a los que posteriormente hemos extraído testigo y roto a compresión en última instancia.

Las curvas obtenidas al interpolar, por mínimos cuadrados, en el diagrama de los puntos experimentales: velocidad de propagación-resistencia, funciones del tipo

$$
\sigma=A_{0} \cdot \exp \left(A_{1} \cdot c_{l}+A_{2}\right)
$$

o bien

$$
\ln \sigma=A c_{l}+B^{*}
$$

en donde $\sigma$ representa la resistencia a compresión, de la forma que se muestra en la figura $3 b$, obtenida a partir de una muestra de 18 probetas pertenecientes a la estructura de una de las obras estudiada por nosotros.

El límite de error cuadrático medio de los resultados obtenidos, al utilizar dicha curva para la predicción de resistencias a partir de las velocidades de propagación ultrasónica fue sólo del $\pm 15 \%$ para un grado de confianza del $95 \%$.

No obstante el gran interés que tienen los estudios sobre la citada dependencia entre la resistencia a compresión y la velocidad de propagación de impulsos ultrasónicos, de momento, y para los fines del presente trabajo, nos interesa destacar únicamente el hecho de que a velocidades próximas a los $1.000 \mathrm{~m} / \mathrm{s}$ corresponden ya resistencias francamente bajas, del orden de los $20-30 \mathrm{kp} / \mathrm{cm}^{2}$.

Por lo tanto, si tenemos dos zonas de hormigón superpuestas de velocidades tan diferentes como $1.000 \mathrm{~m} / \mathrm{s}$ y $4.000 \mathrm{~m} / \mathrm{s}$, resulta fácil deducir el espesor de cada zona, habida cuenta de la longitud total recorrida por el impulso y el tiempo total de tránsito emplea-

* Hemos utilizado funciones exponenciales o logarítmicas para la representación de la correlación velocidad-resistencia, por ser el tipo de función que mejor se adapta a las particularidades de dicha correlación y ser la forma de función propuesta por la mayoría de los investigadores. 


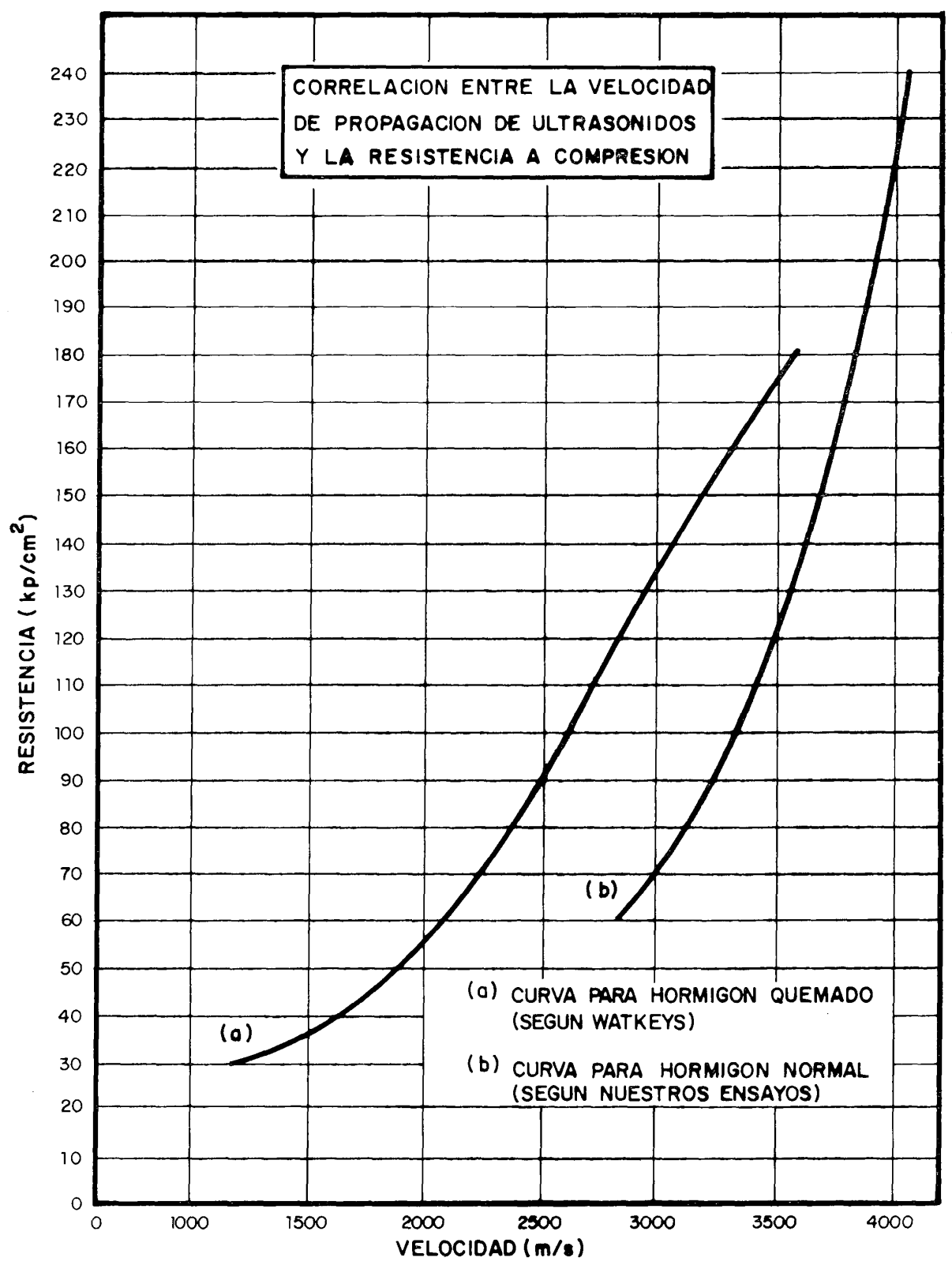

Fig. 3

do por el mismo. Este es, en esencia, el fundamento del método propuesto para la evaluación de los daños profundos ocasionados por la exposición del hormigón al fuego y que habíamos citado al hablar de ello al principio.

Se trata, pues, no de una determinación de la resistencia residual de las piezas de hormigón calcinado mediante correlaciones velocidad-resistencia más o menos discutibles, sino de calcular la parte de camino recorrido por los impulsos ultrasónicos de ensayo en el núcleo interior no calculado de un elemento de hormigón que ha estado expuesto al fuego.

Así, una vez repetido el ensayo según diversas trayectorias que crucen la sección de la pieza en estudio, se podrá fijar, de forma aproximada, el tamaño de la misma y su relación $(\%)$ respecto a la sección total, con lo que podrá conocerse la reducción de sección útil experimentada por un elemento cualquiera a causa de la acción calcinadora del fuego. 
Para ello partimos de las siguientes hipótesis como base:

1. ${ }^{\text {a }}$ El fuego ataca al hormigón de fuera a dentro hasta una cierta profundidad máxima, $P_{M}$, que se puede determinar por diversos ensayos y que dependerá, naturalmente, de la temperatura alcanzada y del tiempo de exposición al fuego.

2. ${ }^{a}$ El gradiente de temperatura a través de toda sección transversal de las piezas calcinadas (viga, pilar, etc.), induce otro gradiente análogo de velocidades de propagación sónica en dicha zona, que suponemos lineal, en primera aproximación, para mayor simplicidad de los cálculos.

3. El hormigón en estudio, antes del siniestro, era lo suficientemente homogéneo como para permitir la consideración de una velocidad media de propagación en el mismo $\bar{c}_{N}$, sin que por ello se cometan errores importantes.

En virtud de la hipótesis $2 .^{\text {a }}$ se puede tomar una velocidad media de propagación $\bar{c}_{Q}$ en toda la parte de sección quemada, lo que equivale a considerar dicha zona como uniformemente calcina. da. Esto que, naturalmente, no es exacto, no importa para el cálculo de longitudes recorridas efectivamente por el impulso si $\bar{c}_{Q}$ es el valor medio de dichas velocidades en las zonas de material calcinado.

Haciendo uso de esta $\bar{c}_{Q}$ y de la velocidad media de propagación $\bar{c}_{N}$ en hormigón sano podemos emprender el siguiente cálculo. 

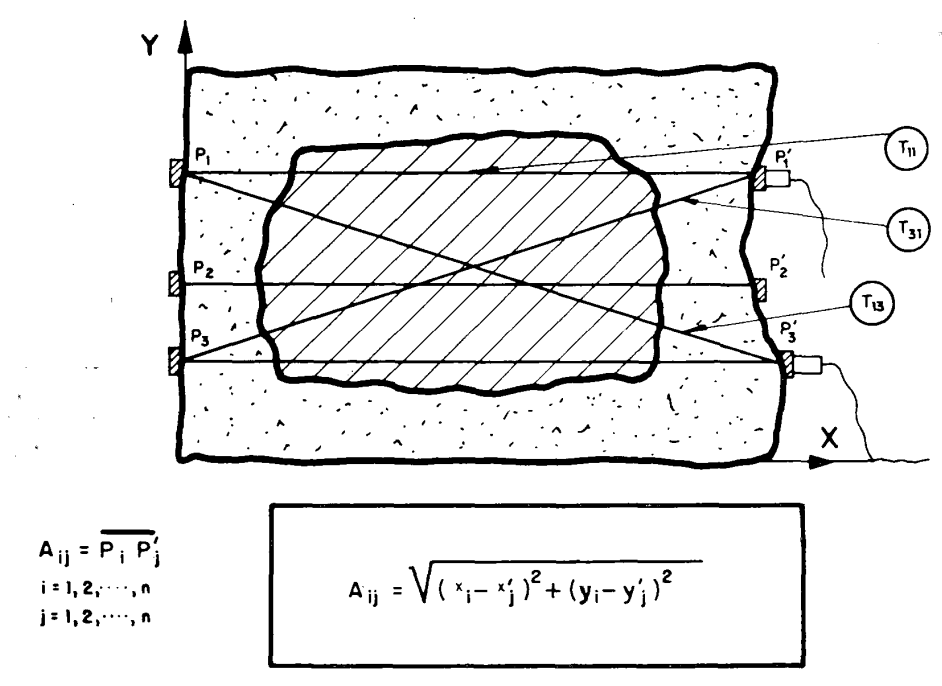

Fig. 6. Trayectorias cruzadas.

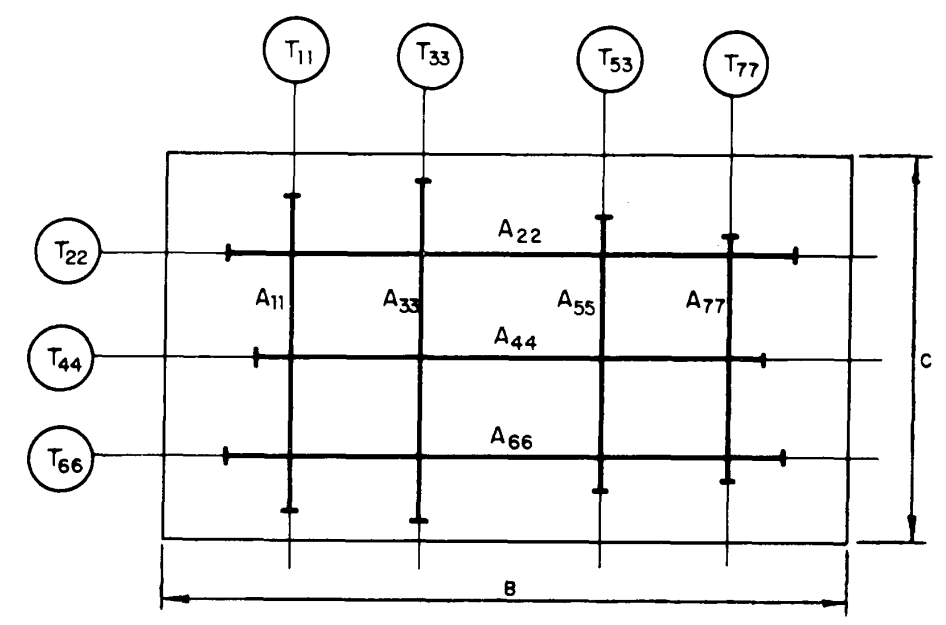

2. CALCUlO DE LA LONGITUD MEDIA ATRAVESADA POR EL HAZ DE ENSAYO EN EL NU. CLEO DE HORMIGON SANO

Sea una sección ortogonal cualquiera de un elemento de hormigón, por ejemplo un pilar parcialmente calcinado (fig. 4). En dicha sección se representa con punteado la zona calcinada, de baja resistencia, en la que la velocidad media de propagación de los impulsos ultrasónicos es $\bar{c}_{Q}$.

Análogamente se representa rayada la parte sana de hormigón correspondiente al nú-

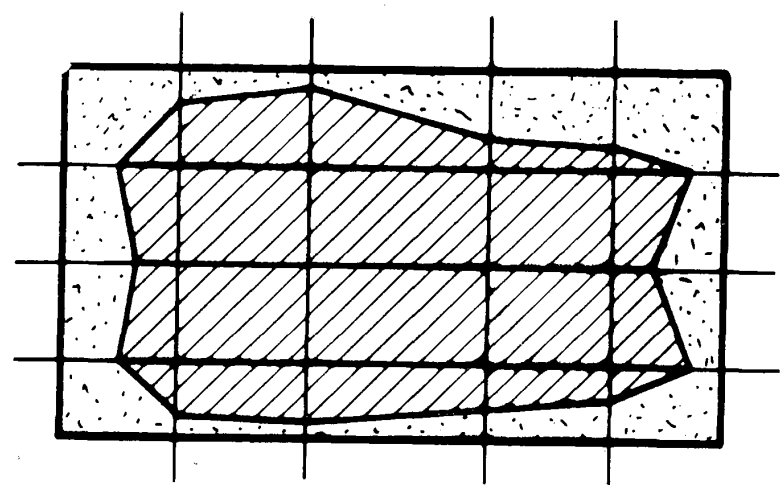
cleo no calcinado del elemento.

Si se envía un impulso ultrasónico a través de dicha sección, siguiendo una trayectoria $T_{i j}$, el tiempo total de tránsito será la suma de los tiempos invertidos en el paso por la zona quemada y por la zona sana. O sea:

$t_{i j}=\frac{L_{i j}-A_{i j}}{\bar{c}_{Q}}+\frac{A_{i j}}{\bar{c}_{N}}$ 


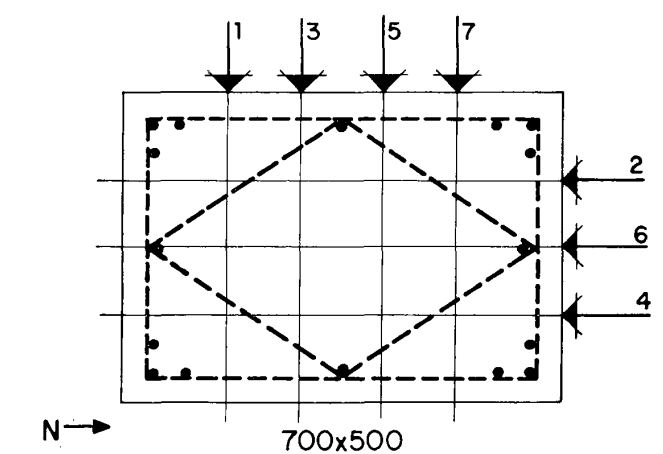

(2)

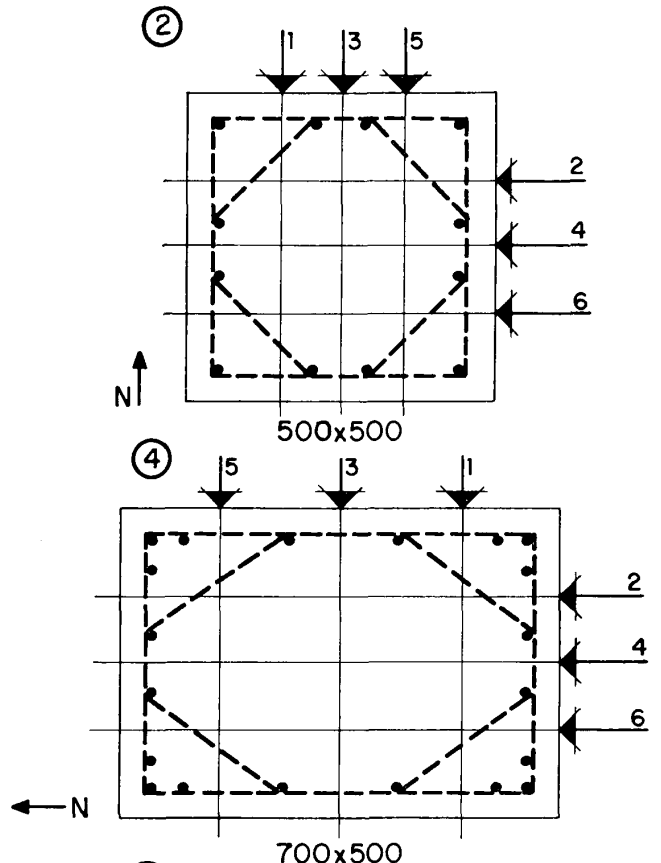

(5)
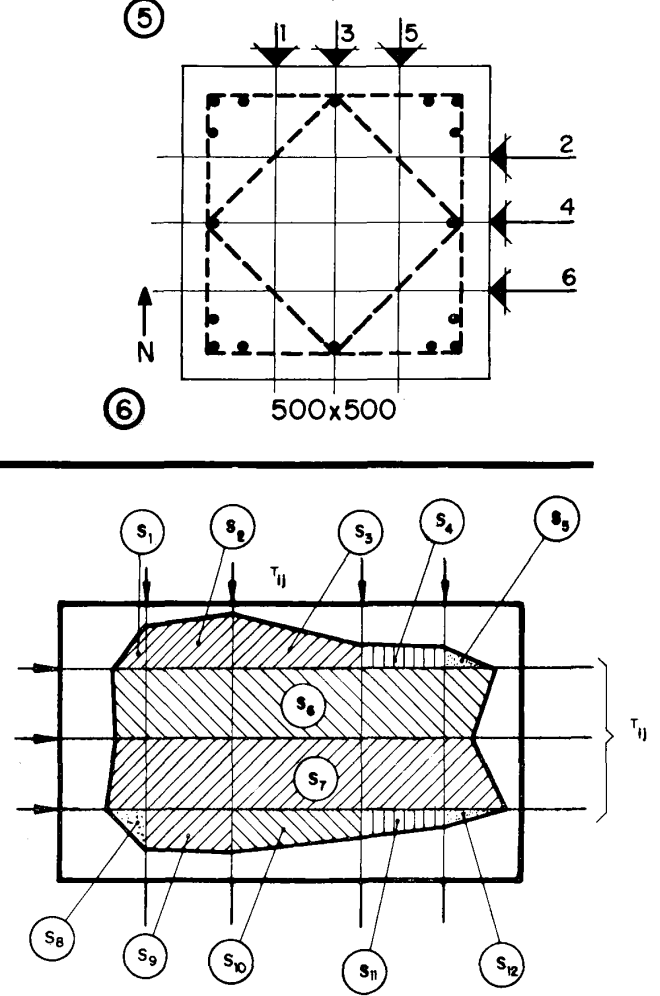

AREA TOTAL $=S=\sum_{i=1}^{i=12} S_{i}$
Donde (fig. 4):

$L_{i j}=$ Longitud total de la sección según la trayectoria considerada $T_{i j}$.

$A_{i j}=$ Segmento de trayectoria, $T_{i j}$, interceptado en el núcleo de hormigón sano y que deseamos calcular.

$t_{i j}=$ Tiempo total de tránsito del impulso de un lado a otro de la sección en ensayo.

Las velocidades $c_{Q}$ y $c_{N}$ tienen el significado anteriormente establecido.

Los subíndices, $i, j$, se refieren a los puntos $P_{i}$ y $P^{\prime}{ }_{j}$ que definen una trayectoria cualquiera $T_{i j}$. Así, si estos puntos se fijan por sus coordenadas en un cierto sistema (fig. 5), se pueden ensayar muchas más trayectorias que las resultantes de enfrentarlos directamente, cruzando unos con otros (fig. 6) y calcular fácilmente las longitudes recorridas por el impulso por:

$$
L_{i j}=\sqrt{\left(x_{i}-x_{j}^{\prime}\right)^{2}+\left(y_{i}-y_{i}^{\prime}\right)^{2}}
$$

Por lo tanto despejando $A_{i j}$ de la fórmula [1], queda:

$$
A_{i j}=\frac{1}{1-\frac{\bar{c}_{Q}}{\bar{c}_{N}}} \cdot L_{i j}\left(1-\frac{\bar{c}_{Q} \cdot t_{i j}}{L_{i j}}\right)
$$

que da la longitud, $A_{i j}$, del núcleo sano según esa trayectoria.

Repitiendo el cálculo para diversas trayectorias, unas paralelas y otras normales a la ya considerada $T_{i j}$ (fig. 7), se obtienen los valores de longitudes correspondientes, $A_{i j}$, que nos permitirán conocer de manera aproximada la forma y dimensiones de la sección de núcleo sano del elemento en estudio.

Para ello, bastará suponer estas longitudes simétricamente colocadas en la sección correspondiente y considerar el polígono de $n$ lados definidos por los extremos de las $A_{i j}$ así dispuestas (fig. 8).

La diversidad de forma y dimensiones de los pilares de la obra siniestrada, así como los diferentes tipos de armado de los mismos, puede conducir a otros tantos sistemas y números de trayectorias de ensayo $T_{i j}$ a emplear, pero los cálculos siguen siendo válidos (fig. 9).

Fig. 9. Trayectorias de exploración en cuatro tipos de pilares según su armadura. Fig. 10. Cálculo del área de una sección de núcleo no 
Se deberá tener en cuenta únicamente, al planear el sistema de trayectorias a emplear, no colocar ninguna de ellas próxima y paralela a alguna armadura o estribo que proporcionaría un camino de alta velocidad para el haz ultrasónico, falseando así la lectura de tiempo correspondiente.

\subsection{Cálculo de la sección del núcleo no calcinado de un pilar}

Una vez calculadas las longitudes $A_{i j}$ correspondientes a una sección dada, se puede calcular su área por descomposición de la figura en triángulos y trapecios (fig. 10), con lo que se llega a expresiones tales como

$$
\begin{aligned}
S=\Sigma S_{i} & =\frac{1}{8}\left[\left(A_{11}+A_{55}\right)\left(A_{22}+A_{66}\right)+\right. \\
& \left.+2 c\left(A_{33}+A_{44}\right)-c^{2}\right]
\end{aligned}
$$

válida para una sección cuadrada y trayectorias de ensayo simétricamente distribuidas (fig. 11).

$\mathrm{O}$ bien

$$
\begin{gathered}
S=\Sigma S_{i}=\frac{1}{8}\left[\left(A_{11}+A_{55}\right)\left(A_{22}+A_{66}\right)+\right. \\
\left.+2\left(b \cdot A_{33}+c \cdot A_{44}\right)-b c\right]
\end{gathered}
$$

si la sección es rectangular con seis trayectorias regularmente distribuidas (fig. 12).

O bien, finalmente,

$$
\begin{gathered}
S=\Sigma S_{i}=\frac{1}{8}\left[c\left(2 A_{66}-2 q-c\right)+b\left(A_{33}+\right.\right. \\
\left.+A_{55}\right)+q\left(3 A_{33}+3 A_{55}-2 A_{11}-2 A_{77}\right)+ \\
\left.+\left(A_{11}+A_{77}\right)\left(A_{22}+A_{44}\right)\right]
\end{gathered}
$$

si la sección es rectangular, e irregular la distribución de las siete trayectorias de ensayo (fig. 13).

Repitiendo esto en varias secciones de un mismo pilar, correspondientes a otros tantos niveles (fig. 14), se podrá tener una idea bas. tante completa del alcance de los daños producidos en el soporte en cuestión.

Por aplicación reiterada del método a la totalidad de los elementos siniestrados se tendrá una visión bastante exacta de la dismi-

\footnotetext{
* Aunque nos estamos refiriendo sólo a pilares, el método es igualmente válido para vigas, con ligeras variantes que no citamos para no hacer demasiado extensa esta exposición.
}
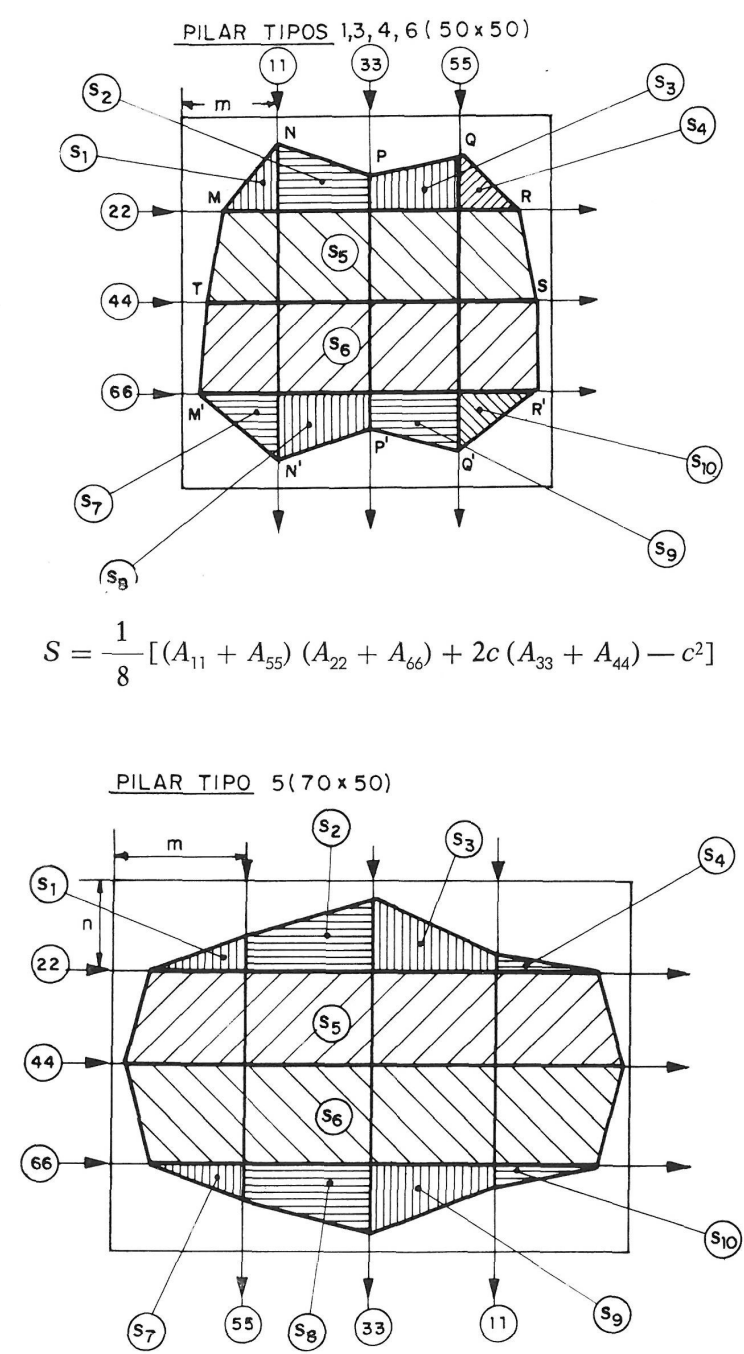

$$
S=\frac{1}{8}\left[\left(A_{11}+A_{55}\right)\left(A_{22}+A_{66}\right)+2\left(b A_{33}+c-A_{44}\right)-b c\right]
$$

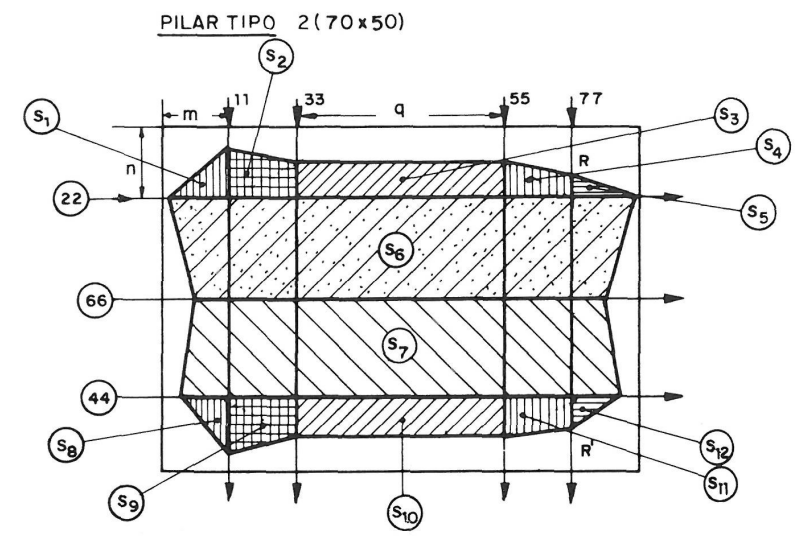

$$
\begin{gathered}
S=\frac{1}{8}\left[c\left(2 A_{66}-2 q-c\right)+b\left(A_{33}+A_{55}\right)+\right. \\
\left.+q\left(3 A_{33}+3 A_{55}-2 A_{11}-2 A_{77}\right)+\left(A_{11}+A_{77}\right) \cdot\left(A_{22}+A_{44}\right)\right]
\end{gathered}
$$

Figs. 11, 12 y 13 . Cálculo del área para tipos concretos de sistemas de trayectorias, $\mathbf{T}_{i j}$. 
nución de resistencia de dichos elementos por pérdida de sección útil a causa de la calcinación sufrida.

Ahora bien, como el volumen de datos a manejar en cualquier incendio real de medianas proporciones es superior al que se podría analizar con éxito de forma manual, se hace preciso el uso de un ordenador, que con un programa adecuado puede calcular las áreas anteriormente indicadas y presentar después los resultados en bloques de cómodo uso e interpretación para el técnico encargado de la reparación de la estructura.

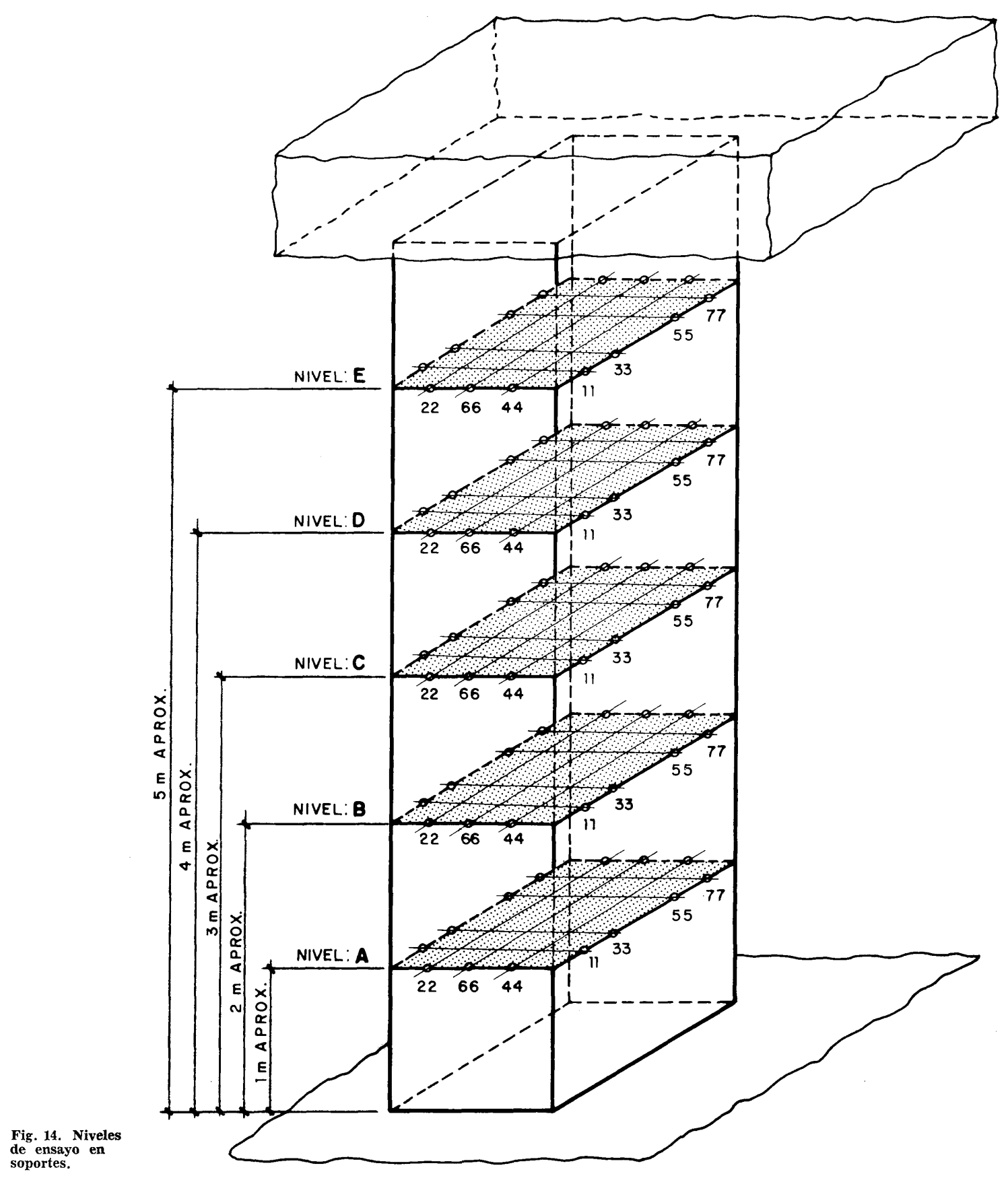




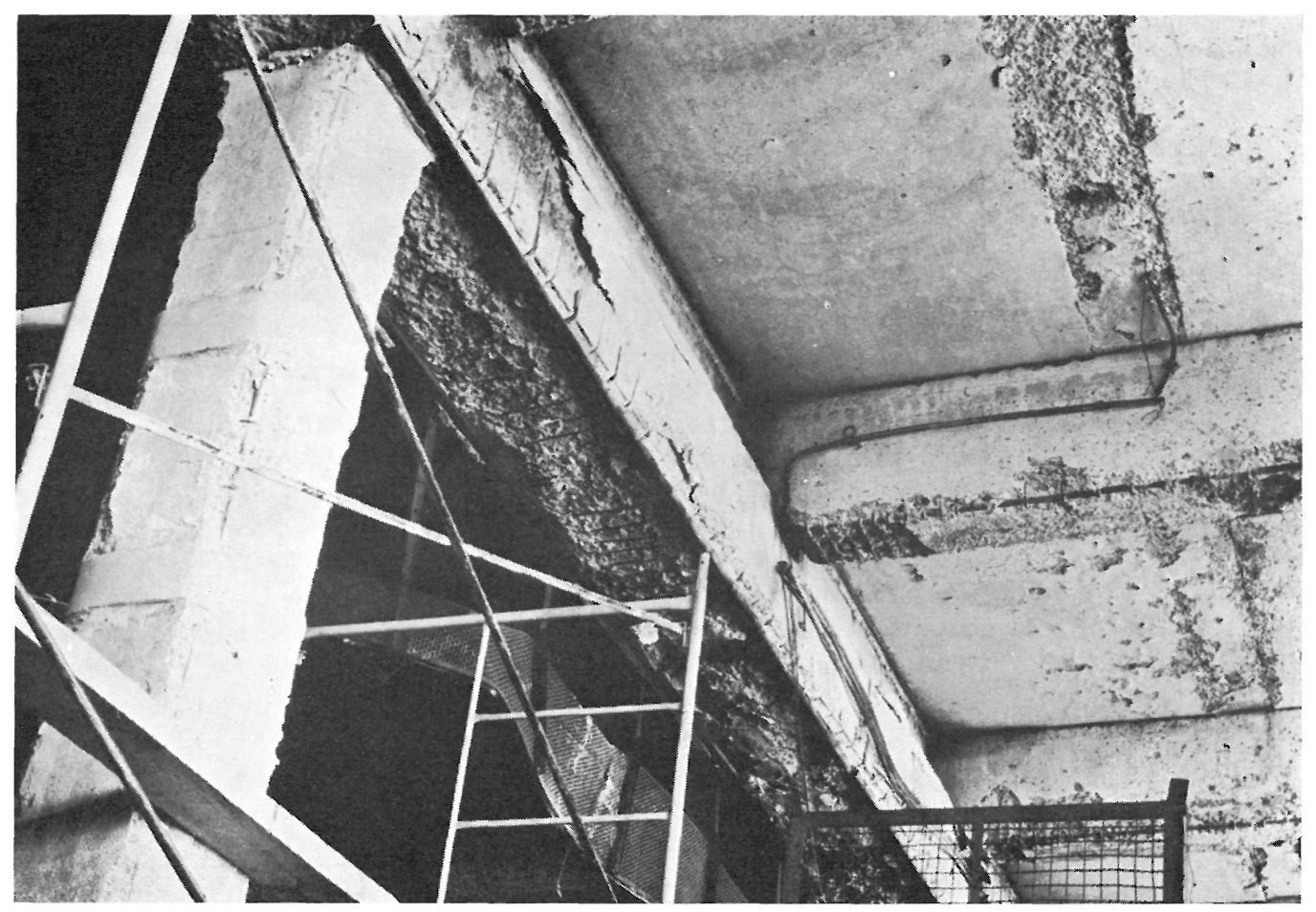

Fig. 15

\section{ENSAYO DEL METODO EN UN CASO REAL}

Como ejemplo vamos a citar el caso del incendio de la nueva factoría de Montaje-2, de Fasa-Renault, en Valladolid, a finales de octubre de 1974 y de cuyo estudio, tras el siniestro, fuimos encargados por la empresa Agromán, S. A., a quien, a su vez, la propiedad había confiado el proyecto y la ejecución de la reparación.

Así, con motivo de este encargo de la citada empresa al Instituto "Eduardo Torroja», de cuya sección de «Ensayos no destructivos e Instrumentación» nos ocupamos, tuvimos ocasión de poner a punto el método de investigación de daños detallado en esta exposición, con resultados francamente satisfactorios.

El incendio, que duró unas 3 horas, afectó a una extensión aproximada de unos $4.000 \mathrm{~m}^{2}$ y los daños fueron cuantiosos.

Las temperaturas alcanzadas debieron estar entre los $700^{\circ} \mathrm{C}$ y $800^{\circ} \mathrm{C}$, según indicios de materiales fundidos.

Se examinaron por el método ultrasónico descrito más de 80 pilares de la zona más afectada, así como 4 vigas principales de $20 \mathrm{~m}$ de luz y una docena de vigas secundarias de $10 \mathrm{~m}$ (fig. 15) *.

La velocidad media en hormigón sano, deducida de ensayos realizados en pilares no afectados por el incendio, así como en las zonas internas de los testigos extraídos de los pilares quemados, fue de:

$$
\bar{c}_{N}=4.200 \mathrm{~m} / \mathrm{s},
$$

lo que indica una buena calidad del hormi. gón inicial.

La velocidad media en hormigón calcinado obtenida de muestras de las capas superficiales más afectadas fue:

$$
\bar{c}_{Q}=1.000 \mathrm{~m} / \mathrm{s} \text {. }
$$

Para confirmar este dato, ya que por el ta. maño y estado de las muestras este número no resultaba muy confiable, se reprodujeron las condiciones aproximadas del incendio (duración, temperatura y posterior choque térmico con agua fría) sobre una serie de

* Las bandas horizontales de yeso que se observan en los pilares son el refrentado que se realizó a posteriori para el ensayo ultrasónico. 
probetas extraídas de un bloque de hormigón de edad análoga al de la estructura siniestrada (fig. 1).

Se midieron las velocidades de propagación ultrasónicas en las probetas, antes y después de su calcinación, con los siguientes resultados (fig. 16).

Aquí puede observarse que en el lote de probetas sometidas a las condiciones presumibles del incendio real $\left(700^{\circ} \mathrm{C}\right.$ y 2 horas de exposición), designadas con M-I en el cuadro de la figura 16, las velocidades de propagación descienden a valores del orden de 1.000 metros/segundo, como ya habíamos dicho.

El programa de cálculo preparado a tal efecto por la División de Cálculo de nuestro Instituto preveía la incorporación de ciertas contingencias en la toma de lecturas, que permitía, no sólo el cálculo de resultados, sino una cierta calificación sobre el valor práctico de los mismos. Así se avisaba, por ejemplo, si el cálculo de una sección se había hecho con un número deficiente de datos (marcado con DD), porque por algún motivo no hubo longitudes $A_{i j}$ suficientes (fig. 17) o porque la zona ensayada estaba muy fisurada (FFF). Igualmente, en los casos que ello ocurría, se advertía que los datos de partida eran muy deficientes (DMD) para el cálculo correcto de la sección. Así, en estos casos, debía entenderse que el valor calculado lo era por defecto y con seguridad mucho menor que el valor real. Asimismo se detectaba una medida anómala en virtud de inscripciones adecuadas insertas en el programa.

De esta manera el técnico responsable, a la vista del informe, podía conocer los resulta-

\begin{tabular}{|c|r|c|c|c|c|}
\hline Muestra & Probeta & $\begin{array}{c}\text { Longitud } \\
(\mathrm{mm})\end{array}$ & $\begin{array}{c}\text { Tiempo } \\
\mathbf{y} \\
\text { temperatura }\end{array}$ & $\begin{array}{c}\mathbf{v}_{\mathrm{O}} \\
(\mathrm{m} / \mathrm{s})\end{array}$ & $\begin{array}{c}\mathbf{V}_{\mathrm{F}} \\
(\mathrm{m} / \mathrm{s})\end{array}$ \\
\hline & 7 & 149 & - & - & - \\
M-I & 8 & 149 & 2 horas & 4.420 & 950 \\
& 10 & 150 & $\mathrm{y}$ & 4.780 & 1.010 \\
& 12 & 148 & $700^{\circ} \mathrm{C}$ & 4.400 & 940 \\
\hline & -3 & 150 & - & 4.700 & 2.170 \\
M-II & 6 & 149 & 3 horas & 4.660 & 2.390 \\
& 11 & 150 & $\mathrm{y}$ & 4.500 & 2.030 \\
& 16 & 150 & $400^{\circ} \mathrm{C}$ & 4.460 & 2.330 \\
& 1 & 149 & - & 4.520 & 4.330 \\
& 2 & 150 & 4 horas & 4.520 & 4.320 \\
M-III & 5 & 150 & $\mathrm{y}$ & 4.640 & 4.430 \\
& 14 & 150 & $150^{\circ} \mathrm{C}$ & 4.640 & 4.490 \\
\hline
\end{tabular}

Fig. 16. Probetas calcinadas (velocidades).

\begin{tabular}{|c|c|c|c|c|c|}
\hline \multirow{2}{*}{ Pilar } & \multirow{2}{*}{ Tipo } & \multirow{2}{*}{ Nivel } & \multicolumn{2}{|c|}{ Sección útil } & \multirow{2}{*}{ Observaciones } \\
\hline & & & $\left(\mathrm{cm}^{2}\right)$ & $(\%)$ & \\
\hline \multirow[t]{3}{*}{$\mathrm{I}-14$} & 2 & B & 1.840 & 53 & DMD \\
\hline & & C & 2.210 & 63 & DD \\
\hline & & $\mathrm{E}$ & & & DI \\
\hline \multirow[t]{5}{*}{$\mathrm{I}-15$} & 2 & A & 3.500 & 100 & 一 \\
\hline & & B & 3.170 & 91 & - \\
\hline & & $\mathrm{C}$ & 3.040 & 87 & - \\
\hline & & $\mathrm{D}$ & 2.230 & 64 & DD \\
\hline & & $\mathrm{E}$ & 3.040 & 87 & \\
\hline \multirow[t]{4}{*}{ I-16a } & 2 & A & 1.750 & 50 & DMD \\
\hline & & B & 1.750 & 50 & DMD \\
\hline & & $\mathrm{D}$ & 1.720 & 49 & DMD \\
\hline & & $\mathrm{E}$ & 1.670 & 48 & DMD \\
\hline \multirow[t]{5}{*}{-16} & 2 & A & 1.750 & 50 & DMD \\
\hline & & B & 1.750 & 50 & DMD \\
\hline & & $\mathrm{C}$ & 1.750 & 48 & DMD \\
\hline & & $\mathrm{D}$ & 1.700 & 48 & FFF \\
\hline & & $\mathrm{E}$ & 1.470 & 42 & FFF \\
\hline \multirow[t]{5}{*}{ I-17 } & 2 & A & 3.170 & 91 & - \\
\hline & & B & 3.170 & 90 & - \\
\hline & & C & 3.120 & 89 & - \\
\hline & & $\mathrm{D}$ & 2.750 & 78 & - \\
\hline & & $\mathrm{E}$ & 2.540 & 72 & - \\
\hline
\end{tabular}

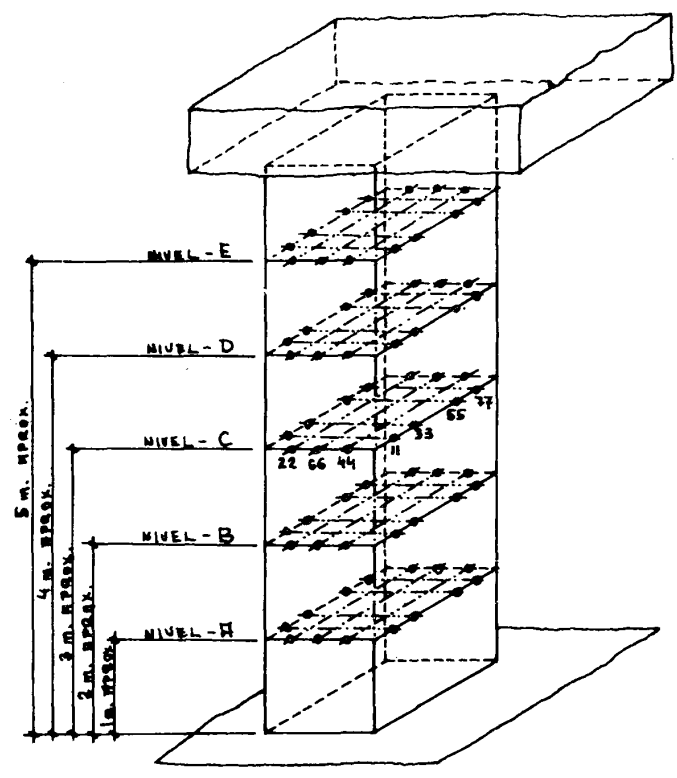

Fig. 1\%. Hoja típica de resultados.

dos fiables, así como los que deberían considerar sólo como valores mínimos de seguridad.

Como ejemplo puede observarse el cuadro de la figura 18, en el que se lee el pilar F-19 (zona de la factoría fuertemente calcinada), en su nivel A (a un metro aproximadamente del suelo), la sección útil es sólo del $38 \%$ de la inicial; pero al estar precedido el valor por la indicación « $\mathrm{DMD}$ » debe entenderse 


\begin{tabular}{|c|c|c|c|c|c|}
\hline \multirow{2}{*}{ Pilar } & \multirow{2}{*}{ Tipo } & \multirow{2}{*}{ Nivel } & \multicolumn{2}{|c|}{ Sección útil } & \multirow{2}{*}{ Observaciones } \\
\hline & & & $\left(\mathrm{cm}^{2}\right)$ & $(\%)$ & \\
\hline \multirow[t]{5}{*}{ F-19 } & 4 & A & 946 & 38 & DMD \\
\hline & & B & 866 & 35 & DMD \\
\hline & & C & - & - & D.I \\
\hline & & D & 728 & 29 & FFF \\
\hline & & E & 一 & - & D.I \\
\hline \multirow[t]{5}{*}{ F-20 } & 4 & A & 625 & 25 & FFF \\
\hline & & B & 625 & 25 & FFF \\
\hline & & C & 625 & 25 & FFF \\
\hline & & D & 625 & 25 & FFF \\
\hline & & E & 652 & 26 & FFF \\
\hline \multirow[t]{5}{*}{ F-21a } & 6 & A & - & 一 & D.I \\
\hline & & B & - & - & D.I \\
\hline & & C & 669 & 27 & DMD \\
\hline & & D & - & - & D.I \\
\hline & & E & - & - & D.I \\
\hline \multirow[t]{2}{*}{$F-21$} & 3 & A & - & - & D.I \\
\hline & & B & - & - & D.I \\
\hline \multirow[t]{3}{*}{ F-23 } & 1 & A & 2.500 & 100 & - \\
\hline & & C & 2.500 & 100 & - \\
\hline & & E & 1.807 & 72 & - \\
\hline
\end{tabular}

Fig. 18. Hoja típica de resultados.

que es valor mínimo de seguridad, ya que no se ha partido en el cálculo de un número completo de datos, y en ausencia de algunos de ellos, el ordenador los ha sustituido por las longitudes mínimas resultantes de la introducción del valor de máxima penetración de daños $P_{M}$.

En otros pilares, como el F-20, las secciones aparecen fuertemente fisuradas (FFF) y, en consecuencia, sólo es posible asegurar el $25 \%$ como sección útil.
Finalmente, en pilares más alejados de la zona de fuego, como el F-23, los primeros niveles aparecen inalterados (sección útil $=$ al $100 \%$ de la sección original) y solamente el último ha resultado algo afectado por el calor, indicando así, que el fuego se había concentrado en las partes altas del mismo junto al forjado.

No cabe duda de que la filosofía del programa se puede afinar cuanto sea posible y destacar en ella los criterios que más nos interesen en cada caso: máxima seguridad, máxima verosimilitud, etc.

Ahora bien, como todo el programa puede ser tratado incluso con miniordenadores de no más de $8 \mathrm{~K}$ de memoria, si se dispone, por ejemplo, de un sistema "Varian-Adapts» de no más de $8 \mathrm{~K}$ de memoria, provisto de pantalla (display), se pueden presentar los resultados de forma visual como si se tratase de una especie de "radiografía» de la sección en estudio, sintetizada numéricamente a partir de los datos de propagación de ultrasonido a su través.

Hasta ahora las experiencias realizadas en este sentido, mediante el empleo del equipo citado y la colaboración para su programación de AUPOCA de España, han sido muy satisfactorias y es de esperar, para un futuro inmediato, que dispongamos de un programa de cálculo completo que incluya las instrucciones pertinentes para la visualización en pantalla de las zonas útiles de las secciones en estudio.

\section{résumé}

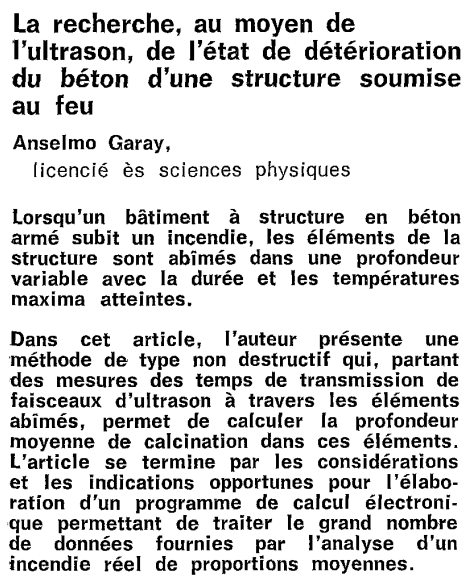

\section{summary}

Supersonic investigation methods regarding deterioration of concrete in structures damaged by fire

Anselmo Garay, M. A. Physics

When a building with a reinforced concrete structure catches fire, the members of that depending upon the duration and maximum depending upon the ouration
temperatures of the fire.

The present article describes a non to calculate the that makes it possible of the structural members by means of measuring the transmission times of ultra sonic beams through the affected elements.

Th article ends proposing suitable considerations and indications for elaborating an electronic calculation programme capable of ing from a fire of average range.

\section{zusammenfassung}

Überschall - Untersuchungsmethoden in Bezug auf Betonschäden einer von Feuer angegriffener Struktur

Anselmo Garay, Liz. Physik

Wenn ein Gebäude mit Stahlbetonstruktur durch Feuer beschädigt wird, werden ihre Teile gemäss der Höchsttemperaturen und der Dauer der Feuer angegriffen.

Der vorhandene Artikel beschreibt eine nicht zerstörende Methode, die es ermöglicht, die Mitteltiefe der Kalzinierung zu berechnen, wobei sie von den übertragungszeiten der Uberschall- Bunndel durch die angegrfif-

Der Artikel präsentiert zum Schluss Angaben und Erwägungen für ein elektronisches $\mathrm{Be}$ echnungsprogramm, das die grosse Datenanzahl einer Analyse eines Feuers
mittelgrossem Umfang behandeln soll. 